\title{
Warum die Hoffnung der Politiker scheitern musste
}

\author{
W. Zimmerli
}

Auf primär einleuchtenden Rat der Gesundheitsökonomen sahen und sehen die Politiker immer noch in der Bettenreduktion, in der Zentralisierung von Infrastruktur und deshalb in der Schliessung von kleinen Bezirksspitälern, um Infrastrukturkosten einzusparen, das Heil zur Gesundung der Gesundheitskosten. Im folgenden soll anhand von Zahlen aus dem Kanton Bern gezeigt werden, dass sich diese Sicht nicht bewahrheitet hat, dass im Gegenteil die Kosten seit diesen Schliessungen übermässig gestiegen sind. Ausserdem sollen die Gründe für das Scheitern gesucht werden.

\section{Ausgangslage}

Von 1998 bis 2000 wurden im Kanton Bern neun kleine Akutspitäler geschlossen oder in Pflegeoder Nachbehandlungsheime umgewandelt. Diese Massnahme sollte dem Gesundheitswesen viele Millionen Franken einsparen. Wie sieht heute die Bilanz aus?

Die Betriebsbeiträge der öffentlichen Hand an die Regional- und Bezirksspitäler, d.h. die Defizite haben sich von 1998 bis 2001 von 192 auf 380 Mio. Franken erhöht. Diese Zahlen haben mich aufgeschreckt und ich habe versucht, an die genauen Zahlen zu kommen, um herauszufinden, wo die Gründe für das Scheitern der Massnahmen zu suchen sind. Von der Gesundheits- und Fürsorgedirektion des Kantons Bern habe ich drei Monate nach meiner Anfrage ein kurzes Mail mit folgender Mitteilung erhalten: Die Unterlagen vor 1999 seien nicht mehr vorhanden und ausserdem seien die Zahlen nicht mehr konsistent, weil ab 1999 die Chronischkranken nicht mehr enthalten seien.

\section{Vorgehen}

Ich habe aus den mir zur Verfügung stehenden Unterlagen des Kantons, die ich anderweitig bekam, einige Zahlen herausgesucht (Abb. 1-7 und 23) und zusätzlich die Jahresberichte von 13 Spitälern meiner Umgebung (2 Regionalspitäler und 11 Bezirksspitäler) für die Jahre 1995 bis 2002, wovon in der erwähnten Zeit 5 Bezirksspitäler geschlossen oder umfunktioniert wurden, auf Exceltabellen ausgewertet (Abb. 8-22). Es handelt sich um folgende Spitäler:

\begin{tabular}{|c|c|}
\hline \multicolumn{2}{|l|}{ Regionalspitäler } \\
\hline \multicolumn{2}{|l|}{ Burgdorf } \\
\hline \multicolumn{2}{|l|}{ Thun } \\
\hline \multicolumn{2}{|l|}{ Bezirksspitäler } \\
\hline Erlenbach & $\begin{array}{l}\text { umfunktioniert } \\
\text { in Nachbehandlung }\end{array}$ \\
\hline \multicolumn{2}{|l|}{ Zweisimmen } \\
\hline \multicolumn{2}{|l|}{ Saanen } \\
\hline Oberdiessbach & umfunktioniert in Pflegeheim \\
\hline \multicolumn{2}{|l|}{ Münsingen } \\
\hline \multicolumn{2}{|l|}{ Belp } \\
\hline Riggisberg & (in Gefahr) \\
\hline Schwarzenburg & geschlossen \\
\hline Langnau & (in Gefahr) \\
\hline Sumiswald & geschlossen \\
\hline Grosshöchstetten & geschlossen \\
\hline
\end{tabular}

\section{Auswertung}

Der Vergleich mit den Kantonszahlen (Abb. 5 und 8) zeigt in etwa die gleichen Fehlbeträge (Betriebsdefizite ist falsch, weil ein verordneter Fehlbetrag kein Defizit ist). Die Angaben in den «Kantonalen Kennzahlen» stimmen nicht immer ganz mit den Jahresberichten überein, aber der Trend ist derselbe. Folgende Veränderungen sind generell und müssen bei der Betrachtung der Diagramme berücksichtigt werden:

- Die Bettenzahl wurde in Bezirks- und Regionalspitälern verringert, was ja zu Einsparungen führen sollte (Abb. 1).

- Die durchschnittliche Aufenthaltsdauer im Spital wurde gesenkt, was wohl eher kostentreibend ist, weil die ersten Tage teuer sind.

- Die Gesamtzahl des Personals blieb in etwa gleich durch Abbau in den Bezirksspitälern und Aufstockung im Zentrum und in den Regionalspitälern (Abb. 2).

- Nur die Zahlen für stationäre und teilstationäre Patienten (Abb. 3 und 4) werden erfasst, während die ambulanten Patienten in fast keiner Statistik erfasst sind. Dies macht eine ganz saubere Auswertung schwierig. Die Zahl der behandelten Patienten blieb im In- 
selspital fast gleich, stieg in den Regionalspitälern wegen der teilstationären Patienten massiv an, ging aber in den Bezirksspitälern wegen des Spitalabbaus und der Schliessungen entsprechend zurück.

\section{Resultate der Auswertung}

- Auf den ersten Blick erscheint die Verdoppelung des Fehlbetrages eindeutig nicht durch die Bezirksspitäler, sondern durch die Regionalspitäler verursacht (Abb. 5 und 8). Die Verdoppelung des Fehlbetrages wird auch bei der Betrachtung der Fehlbeträge der einzelnen Spitäler nicht durch die Bezirks-, sondern durch die Regionalspitäler verursacht (Abb. 9). Nur die Spitäler Münsingen und Langnau haben durch die Übernahme der Patienten von Oberdiessbach und Grosshöchstetten den Fehlbetrag verdoppelt.

- Die Verdoppelung des Fehlbetrages kann auf der Ausgabenseite oder auf der Einnahmenseite liegen (Abb. 6, 11 und 12). Ausserdem muss man bei der Ursachensuche die Werte pro Patient (Abb. 7 und 10) anschauen, auch wenn man weiss, dass die ambulanten Patienten nicht erfasst sind. Man darf aber grob annehmen, dass die ambulanten Patienten prozentual in allen Spitälern ähnlich viel sind.

- Wenn man die Gründe für die Steigerung des Fehlbetrages sucht, sieht man, dass sie bei den Bezirksspitälern auf der Einnahmenseite (Abb. 11) und bei den Regionalspitälern auf der Ausgabenseite liegen (Abb. 12). Der Fehlbetrag wird bei den Bezirksspitälern etwas verringert, weil die Ausgaben in den letzten beiden Jahren zurückgegangen sind.

- Wenn wir nun die Ausgaben nach den drei üblichen Grundsätzen aufschlüsseln und vergleichen, so zeigt sich bei den Aufwendungen pro Patient (Abb. 13) kein Unterschied zwischen den beiden Spitalkategorien, beim Aufwand pro Bett (Abb. 14) liegen die Bezirksspitäler aus verständlichen Gründen deutlich tiefer (kein Poliklinikbetrieb, Notfallstation auf niedrigerem Niveau, keine Intensivpflegestation, weniger ambulante Verpflichtungen usw.). Bei den Ausgaben pro Stelle (Abb. 15) zeigen die Regionalspitäler in den letzten Jahren eine auffällige Steigerung gegenüber den Bezirksspitälern.

- Dadurch wird nun die Frage akut, wo die Ausgabensteigerung bei den Regionalspitälern zustande kommt. Wenn man die Ausgaben der Regionalspitäler aufschlüsselt, wird ersichtlich, dass die beiden Hauptgruppen, wie bekannt, die Personalkosten und die medizinischen Aufwendungen (Operationsmaterial, Medikamente usw.) sind (Abb. 16). Obwohl sich die Verwaltungskosten auch verdoppelt haben, spielt dies aber beim Gesamtaufwand keine grosse Rolle. Die Erhöhung beim Unterhalt ist verhältnismässig. Die übrigen Gruppen (Lebensmittel, Haushalt, Kapitalzinsen und übriges) wurden auch kontrolliert, haben sich aber nicht auffällig vermehrt und fallen ebenfalls kaum ins Gewicht.

- Der Personalaufwand und die medizinischen Aufwendungen werden deshalb aufgeschlüsselt pro behandelten Patienten, pro Bett und pro Stelle (Abb. 17-22). Wir ersehen daraus, dass die Personalkosten pro Patienten fast gleich sind, eher im Bezirksspital etwas höher, aber bei den Regionalspitälern aufgeholt haben. Die Medizinaufwendungen sind nach den Anpassungen an die Spitalschliessungen in den Bezirksspitälern auf gleiches Niveau wie die Regionalspitäler angestiegen, werden aber jetzt wieder deutlich überflügelt, wobei die ambulanten Patienten alles unsicher machen. Pro Bett und pro Stelle schliessen die Bezirksspitäler verständlicherweise besser $a b$, ausser bei den Personalkosten pro Personalstelle, was zeigt, dass das Personalniveau in den Bezirksspitälern nicht schlechter ist.

\section{Verbleibende Fakten}

- Der Fehlbetrag der Regional- und Bezirksspitäler hat sich während der Schliessungen oder Umfunktionierungen von Bezirksspitälern, während des Abbaus von Betten und während der Verkürzung der durchschnittlichen Aufenthaltsdauer der Patienten verdoppelt, statt verringert.

- Der grosse Fehlbetrag ist in den Regionalspitälern durch grosse Mehrausgaben verursacht, in den Bezirksspitälern durch die Mindereinnahmen (Rückstufung Privat Allgemein) leicht gemildert durch kleinere Ausgaben.

- Dies, obwohl die Zahl der behandelten Patienten im Kanton Bern (Abb. 23) nur gering gestiegen ist, vor allem durch die starke Vermehrung der teilstationären Patienten (Abb. 4) der Regionalspitäler und die erzwungene Reduktion in den Bezirksspitälern und dies, obwohl die Zahl des Personals in der Insel leicht und in den Regionalspitälern deutlich (Abb. 2) gestiegen ist, fast ausgeglichen durch den erzwungenen Personalabbau der Bezirksspitäler. 
- Die Mehrausgaben werden durch die beiden Hauptausgabengruppen Personalkosten und Medizinische Kosten verursacht, wobei vor allem die Kosten pro Bett für beide Gruppen massiv in den Regionalspitälern erhöht sind, und ebenfalls die Medizinischen Ausgaben pro Personalstelle.

Die Quintessenz der ganzen Auswertung ist, dass das Schliessen von Bezirksspitälern finanziell nichts gebracht hat und dass im Gegenteil die Teuerung des Gesundheitswesens durch das Spitalwesen in diesen Jahren beschleunigt angestiegen ist.

\section{Gründe für das Scheitern}

Hier betrete ich den Bereich der Spekulationen und der subjektiven, empirischen Werte, was aber nach der Ausbildung in einem grossen Spital, mehreren Weiterbildungen in in- und ausländischen Grosskliniken und fast dreissig Jahren Erfahrung in einem bernischen Bezirksspital einen gewissen Wert beanspruchen darf.

\section{Finanzielle Gründe}

Je grösser ein Spital, desto aufwendiger wird der gleiche Patient behandelt, weil die Abklärungsund Behandlungsinfrastruktur es erfordert.

Weitere Gründe wurden oben erörtert.

\section{Organisatorische, medizinische} und logistische Gründe

- Ein überblickbarer Betrieb arbeitet effizienter und sparsamer.

- Die Patienten sind im Bezirksspital (B.) bekannt und dadurch ist Anamnese, Untersuchung und Therapie an den einzelnen Patienten angepasst und vermeidet Doppelspurigkeiten.

- Die Kommunikation und die Konsiliarwege im B. sind viel rascher. Es gibt keine sinnlosen Wartezeiten.

- Durch die Patientennähe und die individuelle Kontrolle aller Abläufe im B. geschehen weniger Fehler.

- Es werden weniger unnötige Abklärungen und teure Untersuchungen aus Angst vor juristischen Folgen angeordnet.
- Der Chef- oder Oberarzt ist näher am Assistenzarzt und kann korrigierend eingreifen.

- Die Notfälle im B. werden sofort behandelt oder weitergeleitet.

- Das Regional- und Zentrumsspital wird von einfachen Fällen entlastet.

- Das B. hat kein CT, kein MRI und keine komplexen Labormöglichkeiten. Deshalb werden weniger solche Untersuchungen verordnet und der Assistenzarzt lernt und kennt noch die klinische Untersuchung.

- Es gibt kaum nosokomiale Infekte, d.h. spitalbedingte Zusatzerkrankungen im B.

- Bei Behandlung der Patienten der Grundversorgung in Regional- und Zentrumsspital sind diese Zentren überlastet und es kommt zur Überarztung, d.h. zu sinnlos teuren Behandlungen.

\section{Menschliche Gründe}

- Im kleinen ihm vertrauten Hausspital fühlt sich der Patient geborgen, was zu einer rascheren Heilung führt.

- Die Angehörigen sind näher und die Besuche sind weniger umständlich.

- Die Krankenschwester (Pflegefachfrau), die Hilfspflegerinnen und die Reinigungsfrauen kennen die Patienten und tragen zum Abbau der Angst und zum Aufbau der Heilungschancen bei, ebenso die Ärzte.

- Das Vertrauen der Ärzte zueinander lässt Überweisungen einfach und unbürokratisch ablaufen und erlaubt konsiliarische Ratschläge häufig schon am Telefon und ohne Formularverzögerungen.

- Das Verhältnis zu den Hausärzten ist freundschaftlich, vermeidet unnötige Doppeluntersuchungen und und lässt kaum sinnlose Fehlzuweisungen zu.

Die Politiker, Gesundheitsökonomen und vor allem auch die Öffentlichkeit sollten endlich einsehen, dass an sich einleuchtende Massnahmen das Gegenteil bewirkt haben, von der seit 1997 grassierenden Fehleinschätzung ablassen und andere Wege vorschlagen, damit die noch verbleibenden Bezirksspitäler nicht mehr um ihre Zukunft bangen müssten und in Ruhe arbeiten könnten. 


\section{Abbildungen}

Alle zu den Abbildungen gehörenden Zahlen sind bei mir einzusehen.

Abbildung 1

Bettenzahlen Kanton Bern.

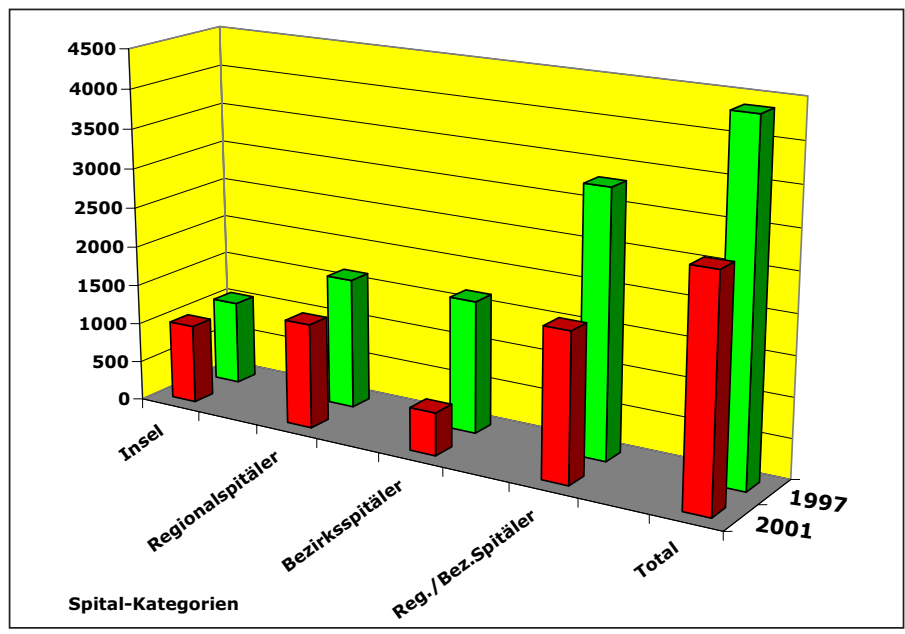

Abbildung 3

Patienten stationär Kanton Bern.

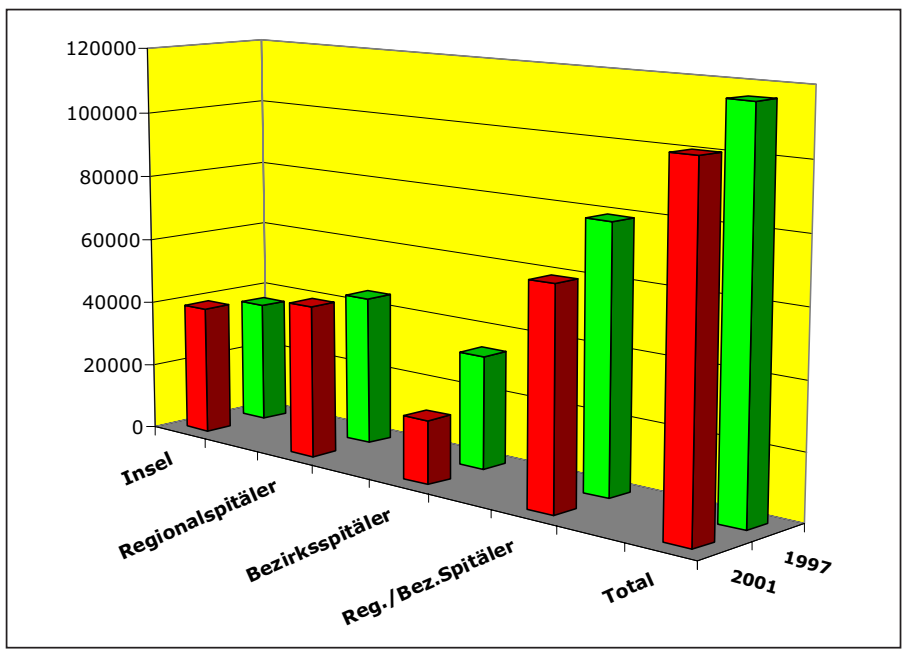

Abbildung 5

Fehlbetrag Spitäler Kanton Bern in Mio. Franken.

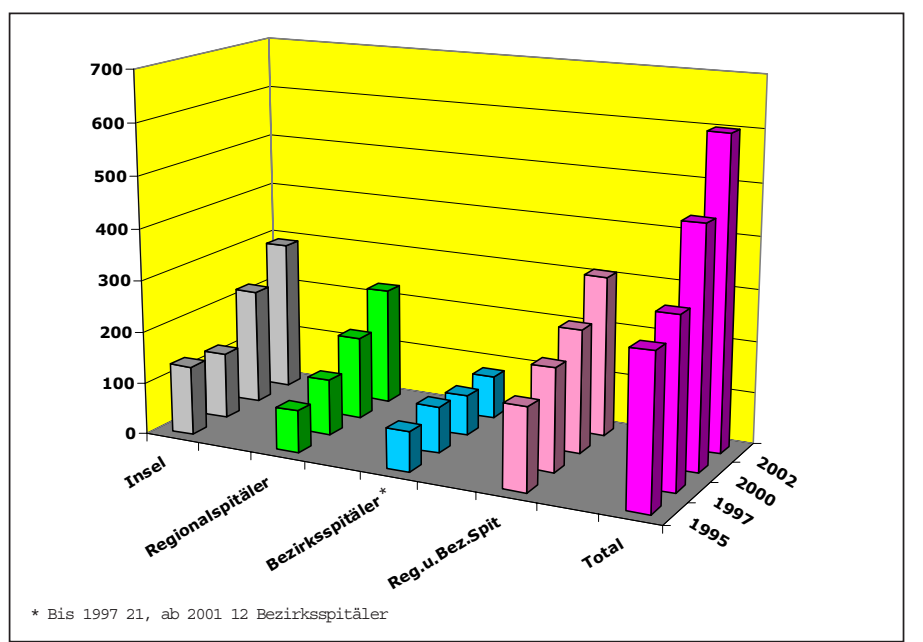

Abbildung 2

Stellen Personal Kanton Bern.

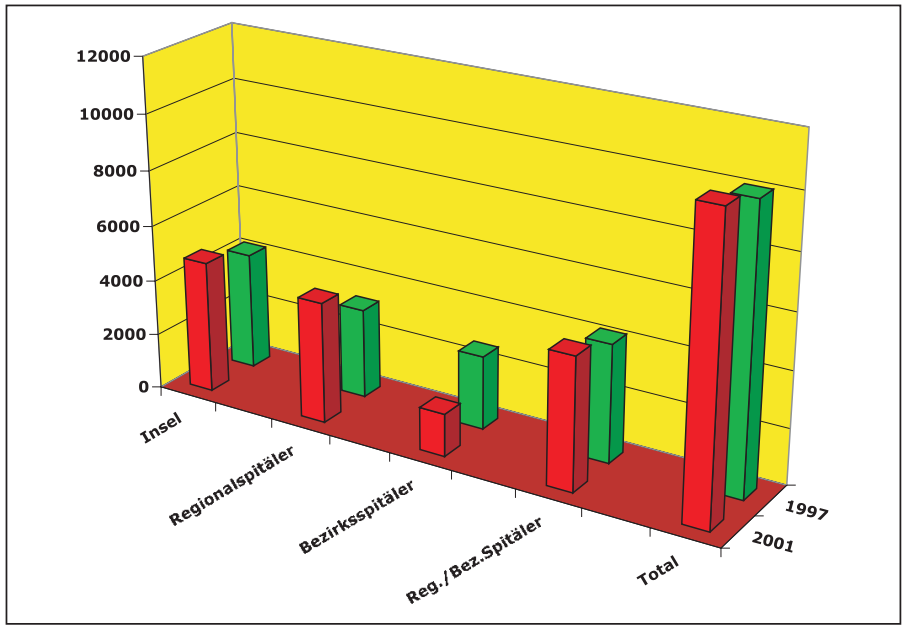

Abbildung 4

Teilstationäre Patienten Kanton Bern.

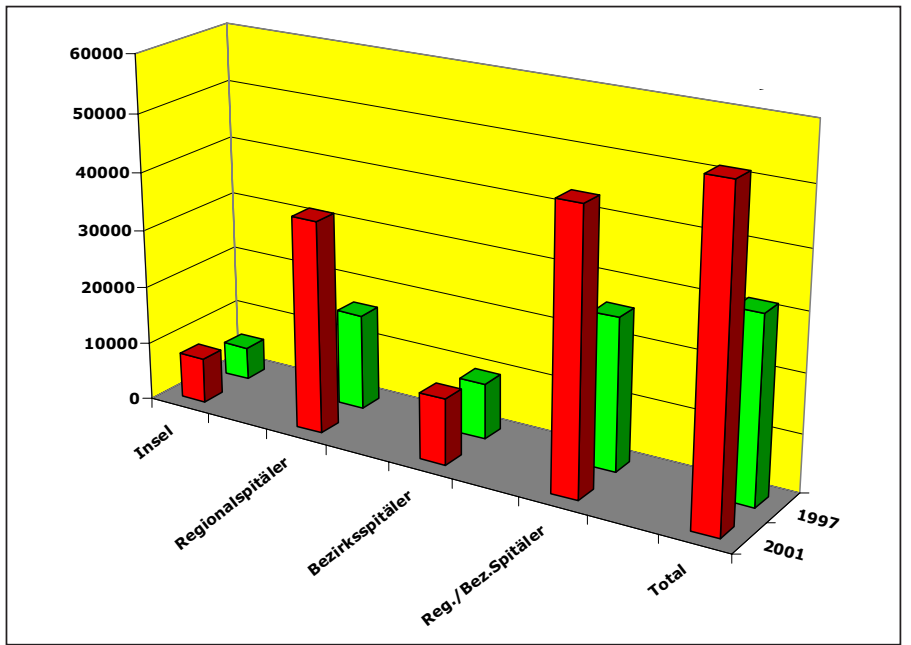

Abbildung 6

Einnahmen, Ausgaben und Fehlbetrag Spitäler Kanton Bern.

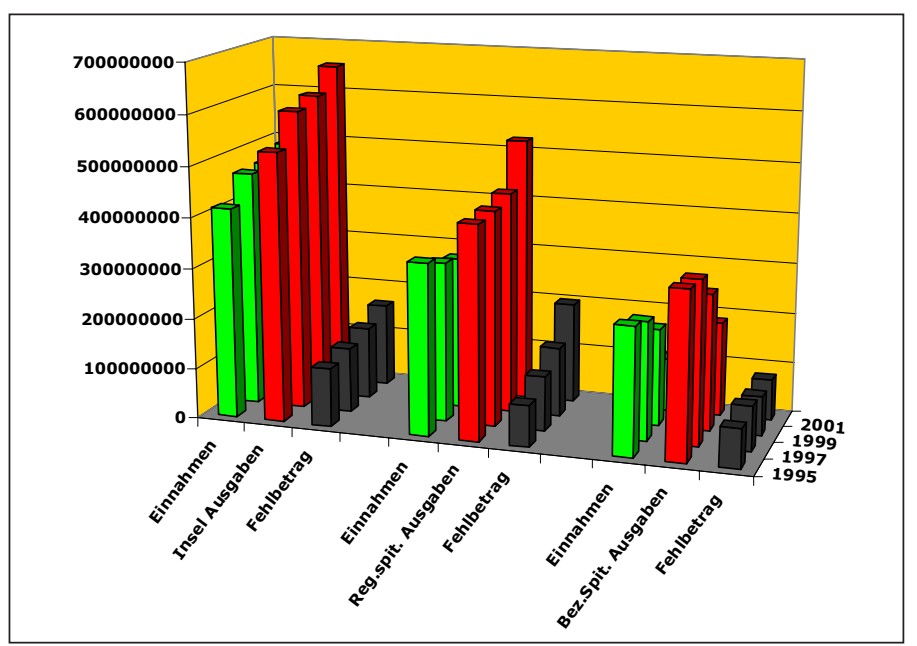


Abbildung 7

Ausgaben, Einnahmen und Fehlbetrag pro Patienten Kanton Bern.

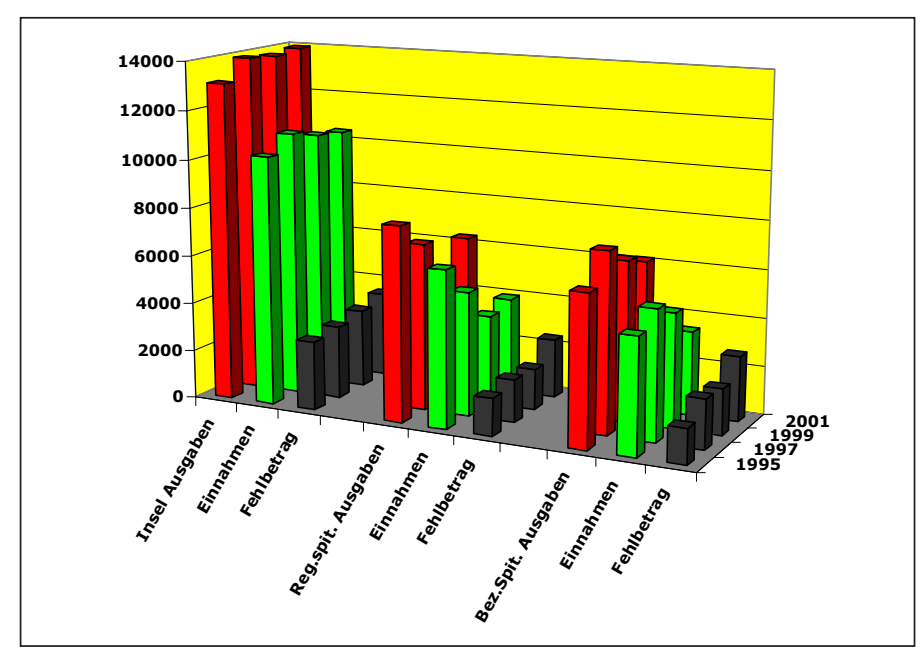

Abbildung 9

Fehlbetrag 13 Spitäler.

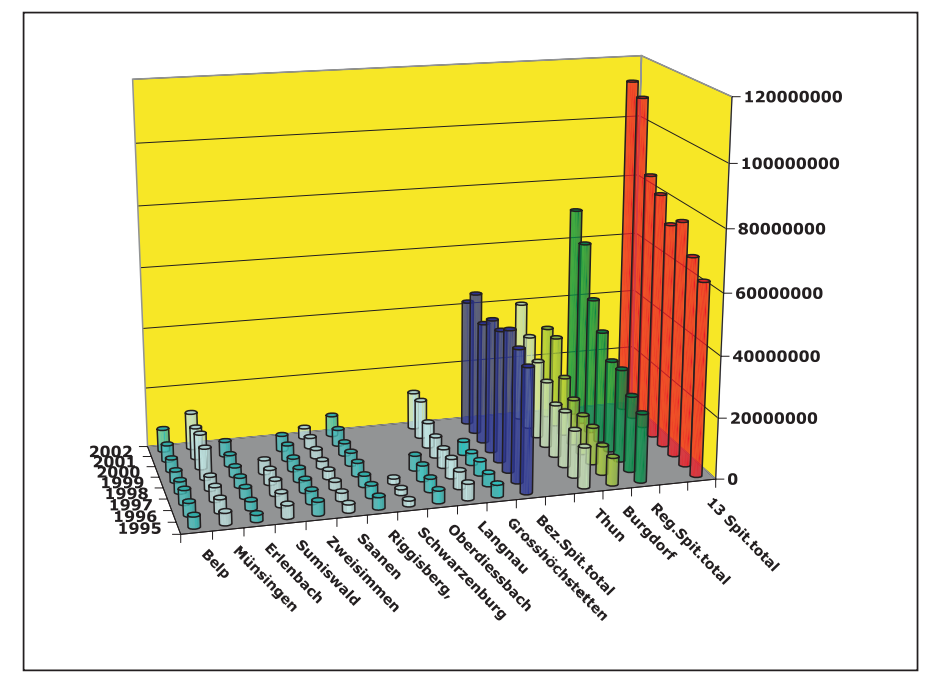

Abbildung 11

Durchschnitt Ausgaben, Einnahmen und Fehlbetrag pro Bezirksspital.

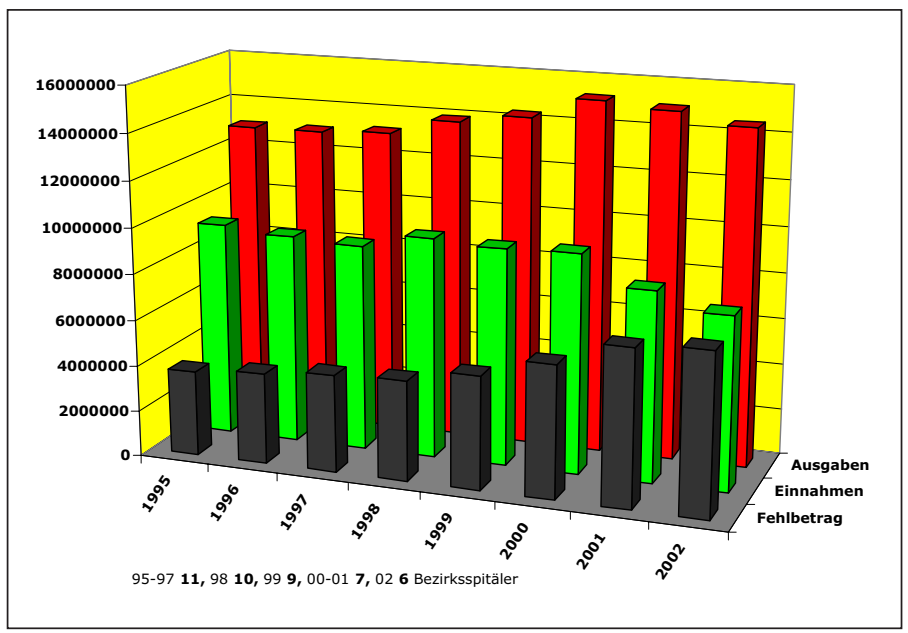

Abbildung 8

Fehlbetrag 13-8 Bezirks- und Regionalspitäler.

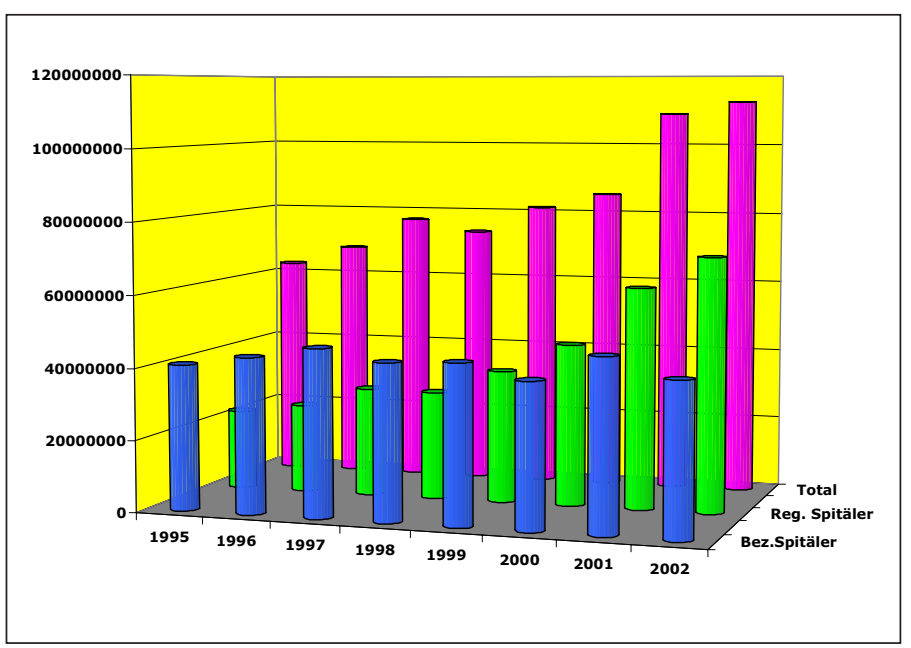

Abbildung 10

Fehlbetrag pro Patienten 13 Spitäler, Regional- und Bezirksspitäler.

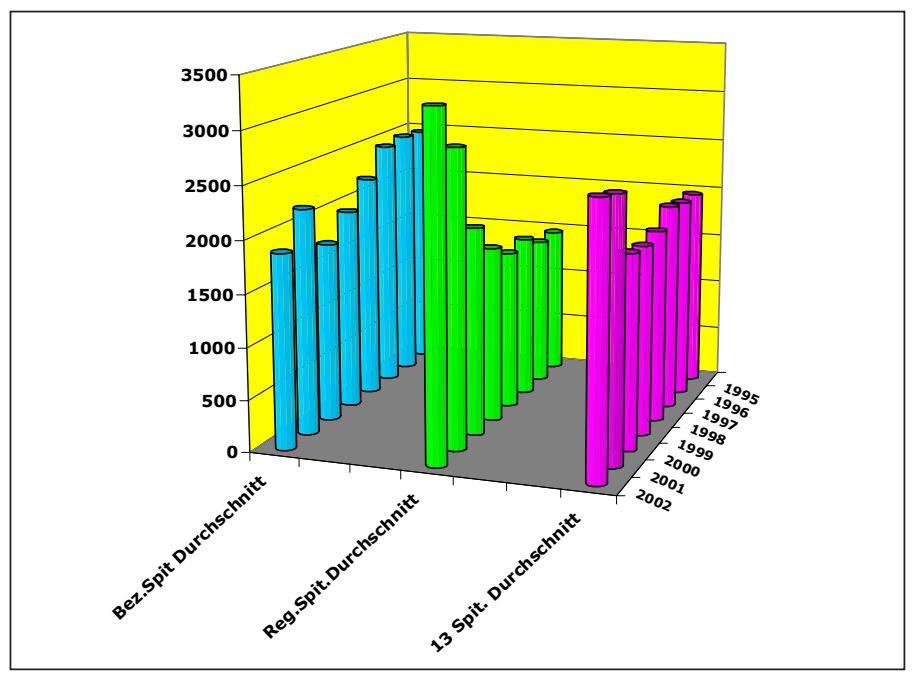

Abbildung 12

Durchschnitt Ausgaben, Einnahmen und Fehlbetrag pro Regionalspital.

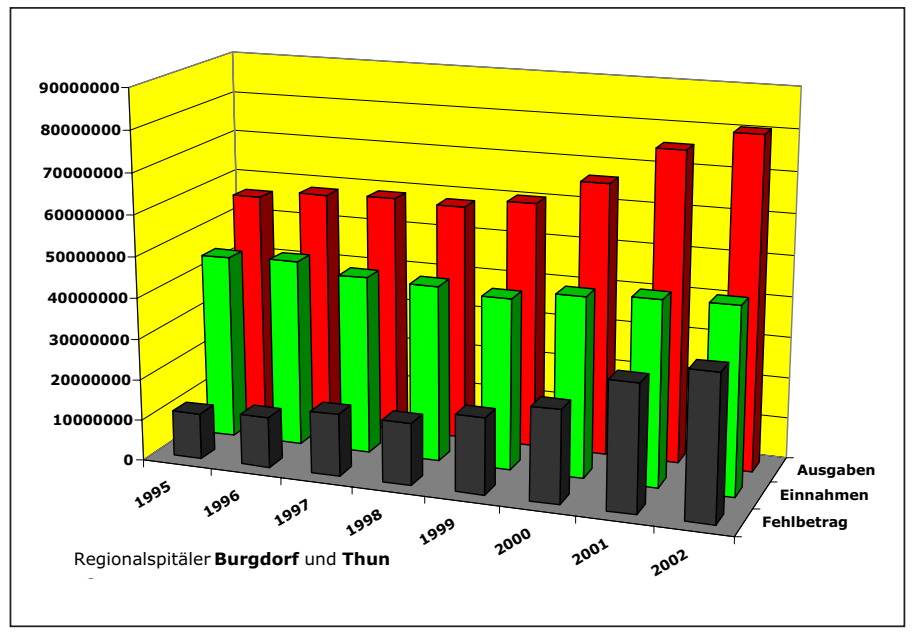


Abbildung 13

Ausgaben pro Patienten 13-9 Spitäler.

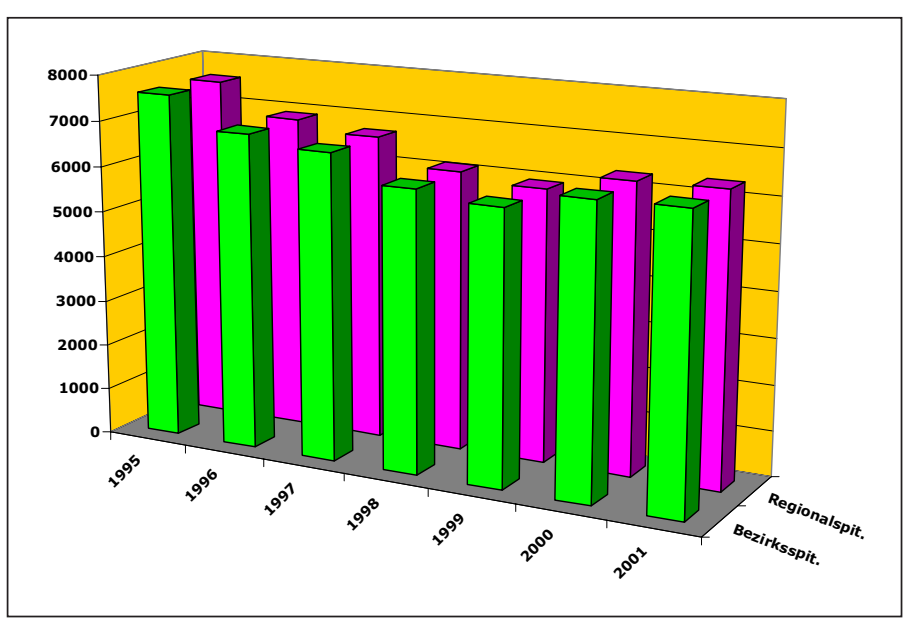

Abbildung 15

Ausgaben pro Stelle.

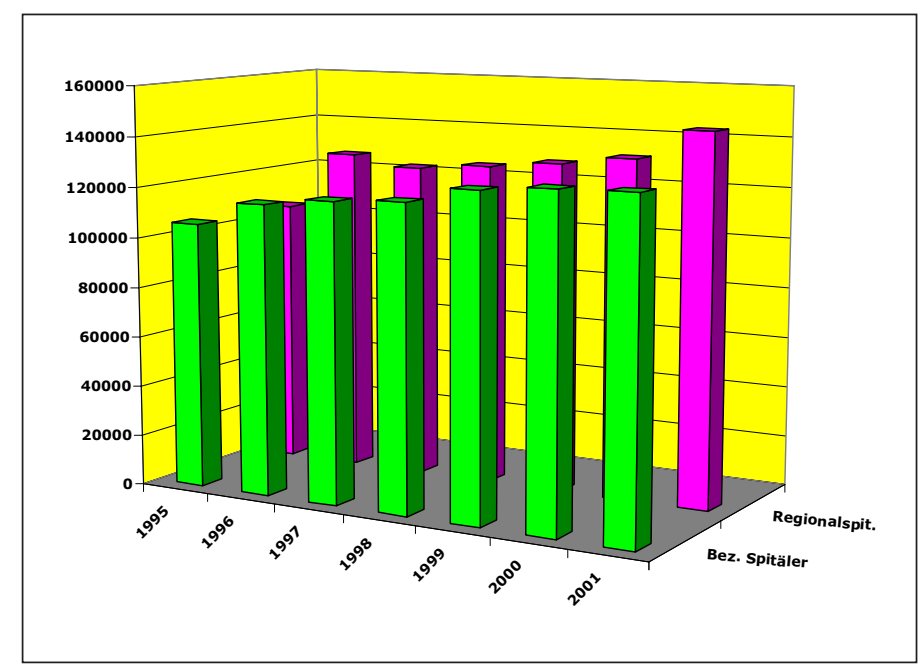

Abbildung 17

Personalkosten pro Patienten.

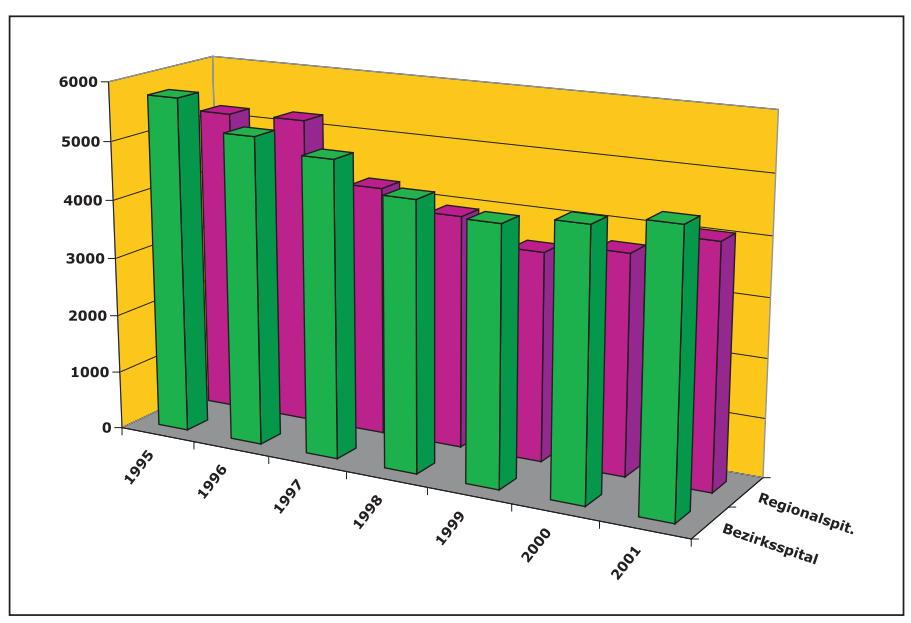

Abbildung 14

Ausgaben pro Bett 13-9 Spitäler.

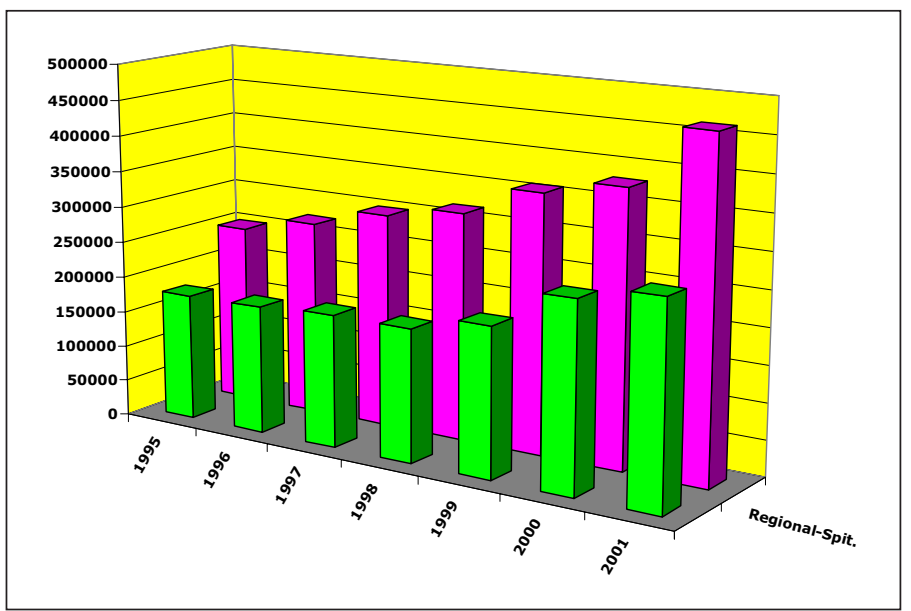

Abbildung 16

Kostengruppen Regionalspitäler Burgdorf und Thun.

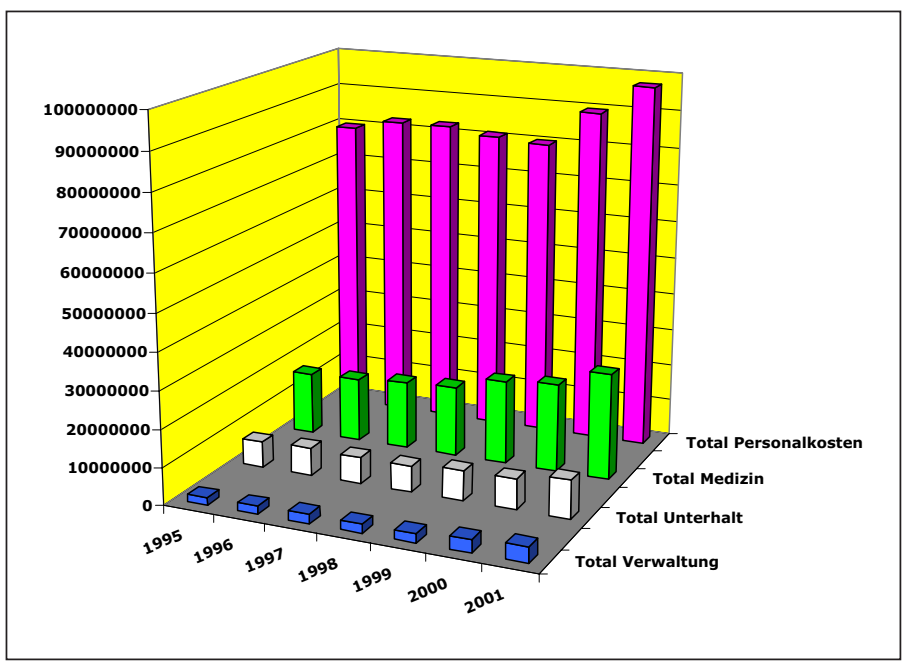

Abbildung 18

Personalkosten pro Bett.

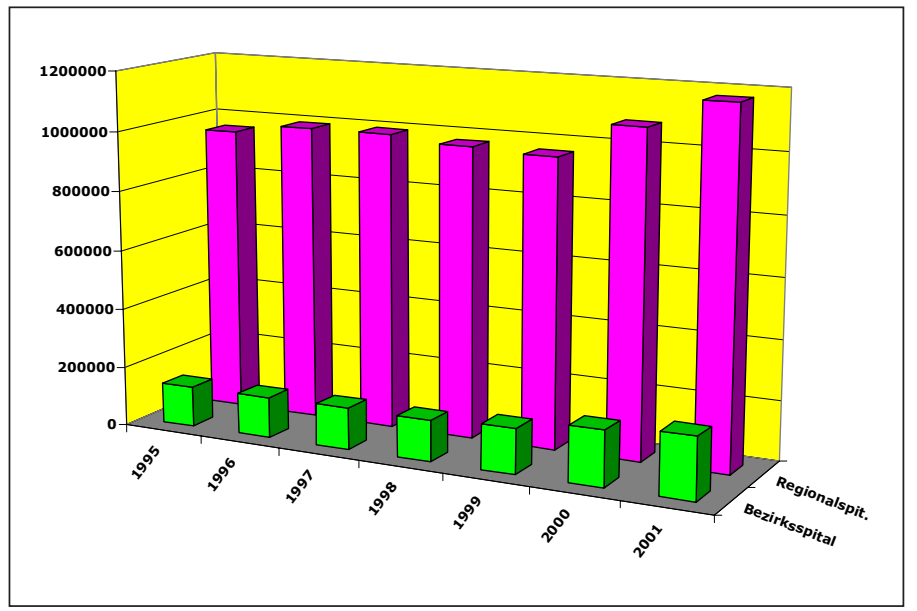


Abbildung 19

Personalkosten pro Personalstelle.

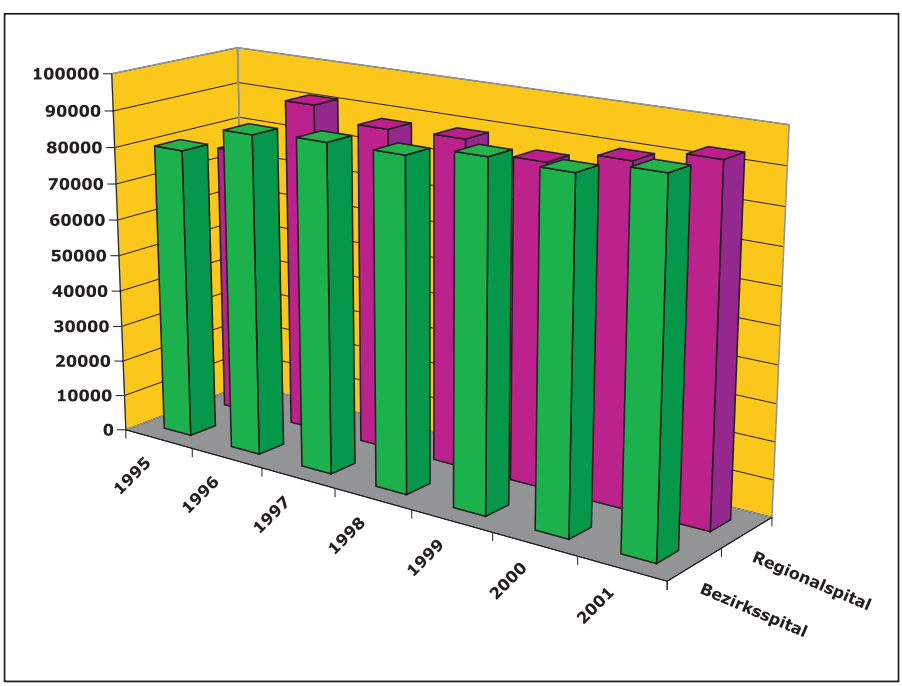

Abbildung 21

Medizinkosten pro Bett 13-8 Spitäler.

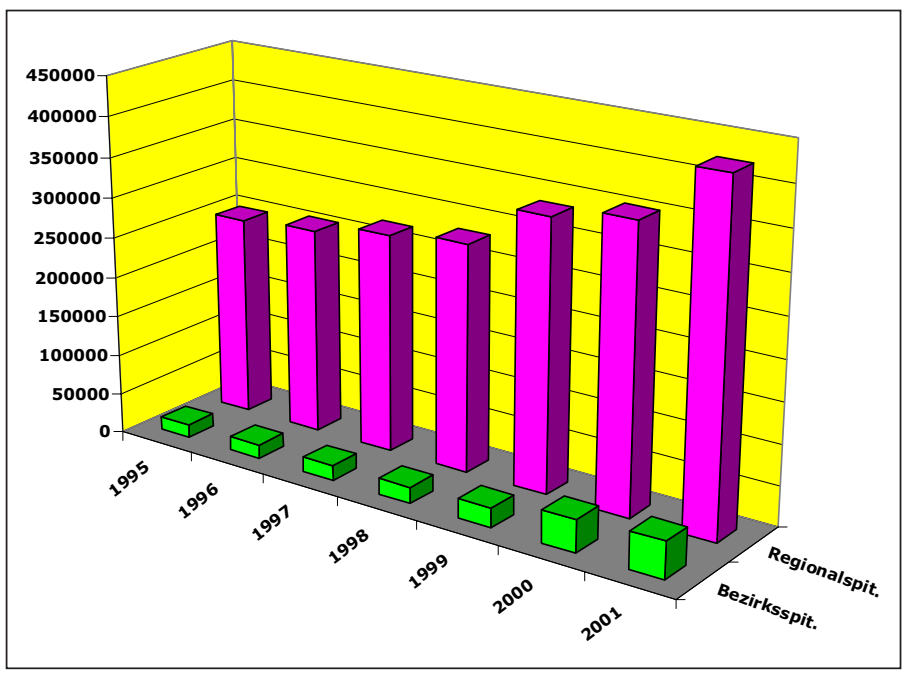

Abbildung 23

Stationär und teilstationär behandelte Patienten im Kanton Bern.

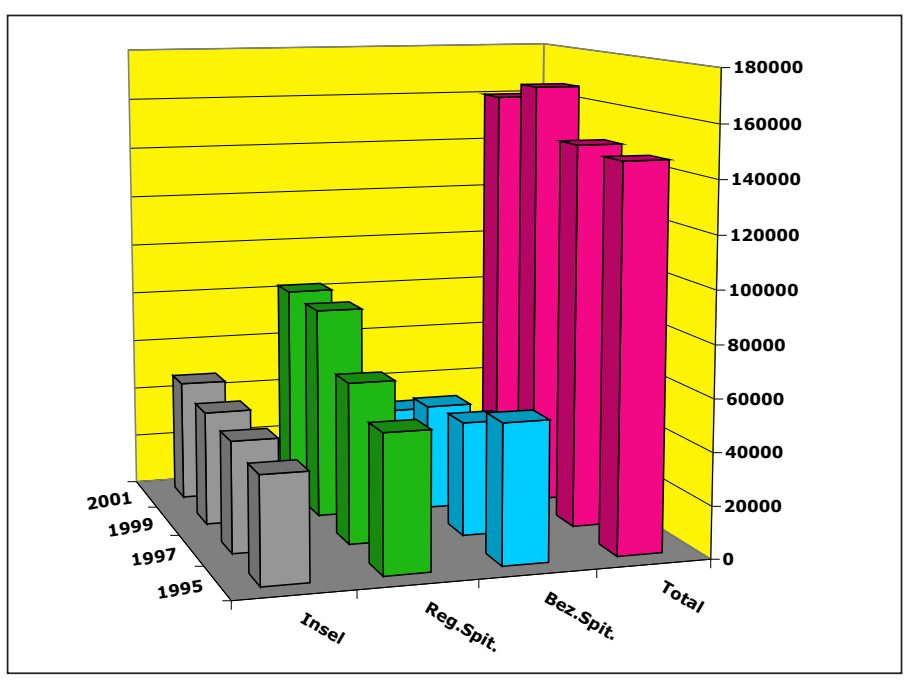

Abbildung 20

Medizinkosten pro Patienten 13-8 Spitäler.

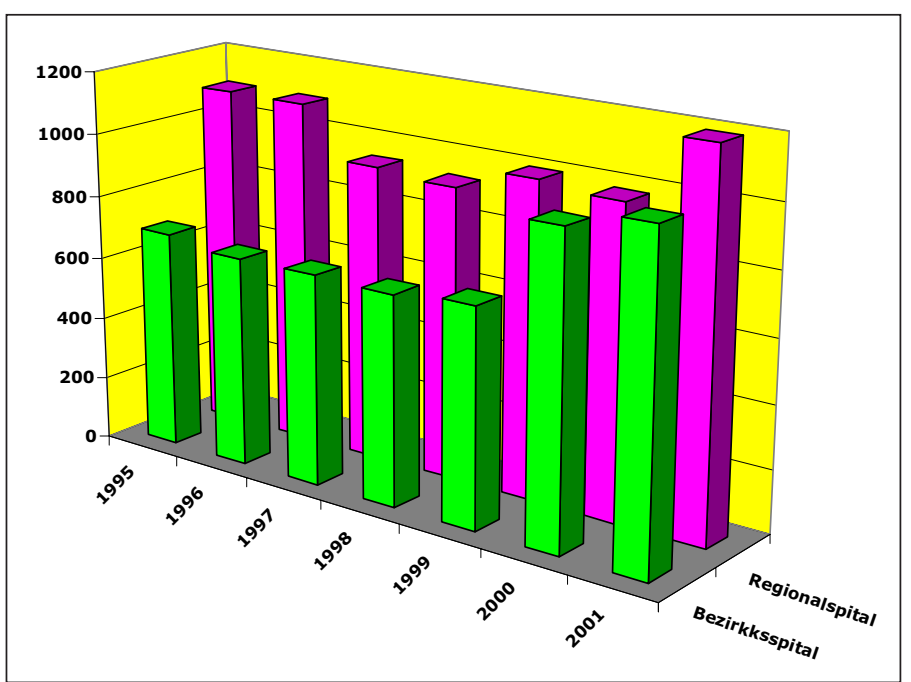

Abbildung 22

Medizinkosten pro Personalstelle 13-9 Spitäler.

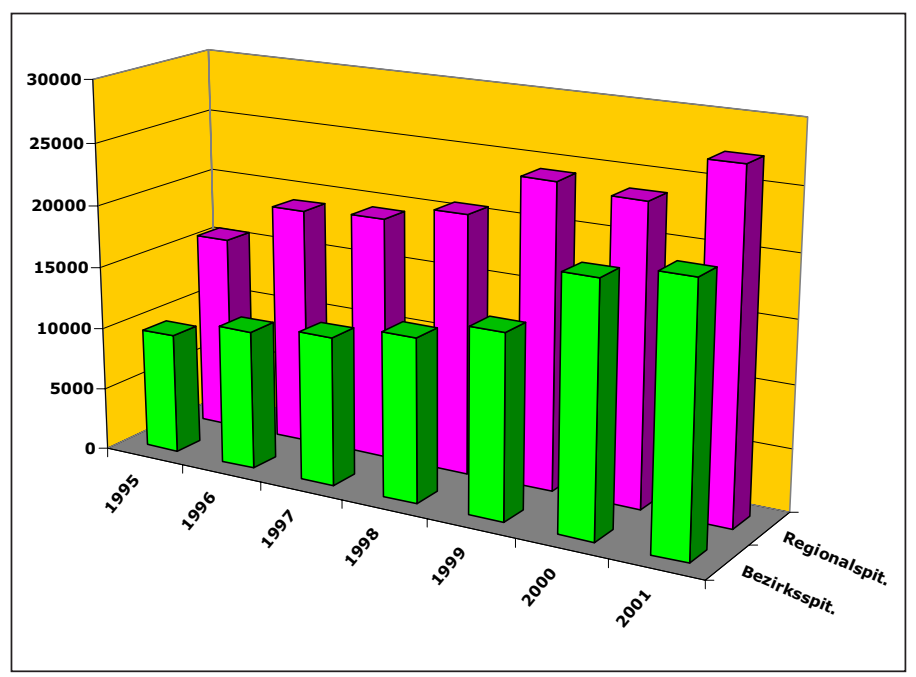

